\title{
Updated ephemeris of Phoebe, ninth satellite of Saturn
}

\author{
N. V. Emelyanov ${ }^{1,2}$ \\ 1 Sternberg astronomical institute, 13 Universitetskij prospect, 119992 Moscow, Russia \\ e-mail: emelia@sai.msu.ru \\ 2 Institut de mécanique céleste et de calcul des éphémérides - Observatoire de Paris, UMR 8028 du CNRS, \\ 77 avenue Denfert-Rochereau, 75014 Paris, France
}

Received 21 May 2007 / Accepted 10 July 2007

\begin{abstract}
Context. The theoretical model of natural satellite motion may need improvement each time new observations of a considerable amount are accumulated over a significant time interval.

Aims. The goal of our work is to elaborate the ephemeris of Phoebe based on all ground-based observations available to date and readily accessible for any user via the Internet.

Methods. Our model for the orbit of Phoebe is based on numerical integration of the equations of motion. In the theory of satellite motion, all necessary perturbations were taken into account. To produce the ephemeris of Phoebe, we used a database that contains all published ground-based observations and expands constantly as new observations appear.

Results. The new ephemeris of Phoebe, based on 1606 ground-based observations made at 39 observatories in the 103 year time interval, was elaborated. The real precision of the ephemeris is limited by the observational precision and is not worse than $0.15^{\prime \prime}$. The ephemeris is available at: http://www.imcce.fr/sat and http://www. sai .msu.ru/neb/nss/index.htm. The ephemeris of Phoebe will be updated as new observations become available.
\end{abstract}

Key words. ephemerides - planets and satellites: general - planets and satellites: individual: Phoebe

\section{Introduction}

The accuracy of the ephemerides of natural satellites decreases as the time elapsed since the period of observation increases. Continuing observations are needed to monitor the ephemerides and, when significant discrepancies occur, an update of ephemerides is needed. Thus, an updated model of satellite motion would be used when it is needed for the production of the ephemeris. Such is the case of natural satellite ephemerides developed at IMCCE (Institut de mécanique céleste et de calcul des éphémérides) on the basis of a similar tool created at SAI (Sternberg Astronomical Institute) (Emelyanov 1996, 2006). At http: //www. imcce. fr/sat you may see our Natural Satellites Ephemeride Server.

The ephemeris of Phoebe, 9th satellite of Saturn, was constantly updated, but was not surveyed in our publications. This paper describes the current state of the ephemeris of Phoebe.

Recent investigations of Phoebe's motion have been based on numerical integration of the equations of motion. The most recent papers have made use of CCD observations for the redetermination of Phoebe's orbit (Arlot et al. 2003; Shen et al. 2005).

\section{Sources of observations and preliminary analysis}

In order to construct the most adequate model of satellite motion, one should take all available observations over the maximum possible time interval. To produce the ephemeris of Phoebe, we used a database of astrometric observations of natural planetary satellites, created at the IMCCE in collaboration with SAI as a part of the Natural Satellite Data Center (NSDC).

This database, which is accessible to any interested person via the Internet (http: //www.imcce.fr/nsdc), contains all published satellite observations, and is constantly expanding as new observations appear; a trilingual version is available at http://www.sai.msu.ru/neb/nss/index.htm. A detailed description of various series of observations, as well as bibliographic references, may be found in the above-mentioned database. The observational basis for the ephemeris of Phoebe is constantly being updated. Currently, all the observations made from 1904 to May 27, 2007, at 42 observatories, are available. The database contains 1660 positions of Phoebe.

During preliminary analysis, 54 observed positions of Phoebe causing residuals in right ascension and declination of more than 2.5 arcsec were rejected. Hence, our ephemeris of Phoebe is based on 1606 observations made at 39 observatories. Statistics representing the observations used for the ephemeris of Phoebe made at different observatories are given in the Table 1.

Since preliminary processing for the observations referred to a mean equator and equinox of an epoch system (other than the FK5/J2000), we first reduced the satellite positions to the FK4/B1950 system with the Newcomb precession constant, and then reduced them to the FK5/J2000 system in accordance with the new IAU resolutions, using the procedure described in Aoki et al. (1983). Finally, the satellite positions were reduced to the ICRF (International Celestial Reference Frame) using the rotation parameters from Feissel \& Mignard (1998). 
Table 1. Residual statistics summary for each observatory ( $N$ pos. - number of satellite positions used).

\begin{tabular}{|c|c|c|c|c|c|c|c|}
\hline $\begin{array}{l}\text { Obs } \\
\text { code } \\
\text { MPC }\end{array}$ & $\begin{array}{l}\text { Observatory } \\
\text { name }\end{array}$ & $\begin{array}{l}\text { Periods of } \\
\text { observation }\end{array}$ & $\begin{array}{c}N \\
\text { pos. }\end{array}$ & $\begin{array}{c}\text { Mean } \\
\frac{r_{\alpha}}{\left({ }^{\prime \prime}\right)}\end{array}$ & $\begin{array}{c}\text { Mean } \\
\overline{r_{\delta}} \\
\left({ }^{\prime \prime}\right)\end{array}$ & $\begin{array}{c}\mathrm{rms} \\
\sigma_{\alpha} \\
\left({ }^{\prime \prime}\right)\end{array}$ & $\begin{array}{c}\mathrm{rms} \\
\sigma_{\delta} \\
\left({ }^{\prime \prime}\right)\end{array}$ \\
\hline 0 & Greenwich & $1907-1910$ & 51 & 0.60 & 0.22 & 1.00 & 0.78 \\
\hline 74 & Boyden Observatory, Bloemfontein & 1957 & 8 & -0.38 & 0.40 & 0.74 & 0.77 \\
\hline 133 & Les Tardieux & 1998 & 12 & -0.20 & -0.14 & 0.44 & 0.43 \\
\hline 143 & Gnosca & 2006 & 3 & -0.24 & -0.10 & 0.25 & 0.16 \\
\hline 185 & Observatoire Astronomique Jurassien-Vicques & 2007 & 5 & -0.04 & -0.76 & 0.23 & 0.90 \\
\hline 215 & Buchloe & 2005 & 3 & 0.03 & -0.05 & 0.10 & 0.13 \\
\hline 286 & Yunnan Observatory & $2003-2005$ & 210 & -0.14 & 0.03 & 0.32 & 0.06 \\
\hline 291 & LPL/Spacewatch II & 2003 & 3 & -0.46 & 0.16 & 0.49 & 0.17 \\
\hline 415 & Kambah, near Canberra & 2004-2007 & 259 & 0.15 & 0.05 & 0.30 & 0.26 \\
\hline 511 & Haute Provence & 1998-1999 & 163 & 0.05 & 0.15 & 0.16 & 0.24 \\
\hline 566 & Haleakala-NEAT/GEODSS & 1996 & 3 & -0.11 & -0.11 & 0.14 & 0.11 \\
\hline 568 & Mauna Kea & 2004-2006 & 9 & 0.11 & 0.16 & 0.37 & 0.49 \\
\hline 644 & Palomar Mountain/NEAT & 2001 & 3 & 0.10 & -0.05 & 0.20 & 0.24 \\
\hline 662 & Lick Obs., Mount Hamilton & 1904-1908 & 19 & -0.46 & -0.47 & 1.05 & 0.89 \\
\hline 672 & Mount Wilson & 1940 & 1 & -1.01 & -0.77 & - & - \\
\hline 673 & Table Mountain & 2001-2005 & 116 & 0.02 & -0.05 & 0.13 & 0.15 \\
\hline 675 & Palomar Mountain & 1994, 1999 & 11 & -0.13 & -0.21 & 0.35 & 0.34 \\
\hline 688 & Lowell Observatory, Anderson Mesa Station & 1981 & 8 & 0.49 & -1.51 & 0.90 & 1.60 \\
\hline \multirow[t]{2}{*}{689} & U.S.N.O., Flagstaff & $1960,1998-2005$ & 187 & 0.01 & -0.03 & 0.26 & 0.31 \\
\hline & & $2005-2006$ & 29 & 0.20 & -0.20 & 0.45 & 0.47 \\
\hline 691 & Steward Observatory, Kitt Peak & $2000-2007$ & 21 & 0.11 & 0.17 & 0.22 & 0.25 \\
\hline 695 & Kitt Peak & 1969,2000 & 4 & -0.23 & 0.48 & 0.25 & 0.54 \\
\hline 696 & Whipple Obs., Mt. Hopkins & 2000 & 3 & -0.29 & -0.01 & 0.32 & 0.15 \\
\hline 703 & Catalina Sky Survey & 2000 & 4 & -0.09 & 0.22 & 0.43 & 0.33 \\
\hline 704 & Lincoln Lab., NewMexico & $1998-2007$ & 148 & 0.15 & 0.35 & 0.68 & 0.88 \\
\hline 711 & McDonald Obs., Fort Davis & $1942,1955,1975-1976$ & 16 & 0.74 & -0.44 & 0.96 & 0.76 \\
\hline 754 & Yerkes Obs., Williams Bay & $1912,1913,1955$ & 13 & -0.12 & -0.62 & 0.86 & 0.83 \\
\hline 761 & Zephyrhills & 2001 & 3 & -0.39 & -0.14 & 0.44 & 0.33 \\
\hline 809 & ESO, La Silla & $1981,1982,1995-2000$ & 63 & 0.39 & -0.16 & 0.76 & 0.69 \\
\hline 822 & Cordoba & 1952 & 7 & -0.68 & -0.80 & 1.16 & 0.86 \\
\hline 874 & Itajuba & 1995-1997 & 60 & -0.05 & 0.20 & 0.18 & 0.36 \\
\hline 950 & La Palma & 1995-1997 & 7 & -0.03 & 0.02 & 0.10 & 0.16 \\
\hline 958 & Observatoire de Dax & 2007 & 6 & 0.08 & 0.13 & 0.13 & 0.22 \\
\hline 999 & Bordeaux-Floirac & 1989 & 5 & 0.23 & -0.22 & 0.27 & 0.31 \\
\hline B01 & Taunus Observatory, Frankfurt & 2007 & 3 & 0.15 & 0.03 & 0.16 & 0.03 \\
\hline B18 & Terskol & 1998 & 9 & -0.02 & -0.11 & 0.07 & 0.14 \\
\hline E12 & Siding Spring Survey & 2006 & 2 & 0.14 & -0.04 & 0.14 & 0.08 \\
\hline G96 & Mt. Lemmon Survey & 2006 & 12 & 0.05 & -0.18 & 0.16 & 0.28 \\
\hline H06 & New Mexico Skies Observatory & 2006 & 2 & 0.14 & 0.14 & 0.14 & 0.14 \\
\hline 337 & Sheshan Station & 2003-2004 & 115 & 0.01 & -0.02 & 0.10 & 0.15 \\
\hline
\end{tabular}

No reductions were made for the most recent observations available in the ICRF. The planetary ICRF positions are calculated from the JPL planetary ephemerides DE405. Thus, Phoebe's motion is modeled in the ICRF.

\section{Orbital motion model}

Our model for the orbit of Phoebe is based on numerical integration of the equations of motion. The method of integration and the fit of the orbital parameters to the observations are the same as described in our paper (Emelyanov 2005). The equations of motion include perturbations from the Sun, Jupiter, Uranus, and Neptune, and the effects of an oblate Saturn ( $J 2$ and $J 4$ only).

The mass of the Sun was augmented by the masses of Mercury, Venus, the Earth-Moon system and the Martian system to take into account part of the perturbing effects from the inner planets.

In our model, the main satellites (Mimas to Iapetus) were replaced by uniform circular equatorial rings. The mass of Saturn is augmented by the mass of the main satellites, and corrected values of $J 2^{\prime}$ and $J 4^{\prime}$ are used to represent perturbations from the main satellites as described in Emelyanov (2005).

The positions of the Sun, Jupiter, Uranus and Neptune are calculated using JPL planetary ephemerides DE405.

Values of the involved parameters were taken from Jacobson (2004) and Jacobson et al. (2006).

\section{Fit to observations and analysis}

The numerical integration of the equations of motion and of the partial derivatives, the fit of orbits to observations, and the representation of satellite coordinates with Chebyshev polynomials, were made using original software.

To integrate the differential equations of satellite motion, we used the method of Belikov (1993). Although this is not necessary in the case of Phoebe, integration was carried out with the variable step, so that the software could select the optimum step.

During the integration process, the series in terms of the Chebyshev coefficients were built representing satellite's rectangular coordinates. The approximation interval was chosen to be 20 days, while the polynomial degree was 13 . Integration of 
Table 2. Residual statistics summary in total.

\begin{tabular}{rcc}
\hline \hline $\begin{array}{r}\text { Type of residual } \\
\text { statistics }\end{array}$ & $\begin{array}{c}\text { In right ascension } \\
\left({ }^{\prime \prime}\right)\end{array}$ & $\begin{array}{c}\text { In declination } \\
\left({ }^{\prime \prime}\right)\end{array}$ \\
\hline Mean value & 0.058 & 0.027 \\
Unweighted rms & 0.436 & 0.447 \\
Weighted rms & 0.231 & 0.228 \\
\hline
\end{tabular}

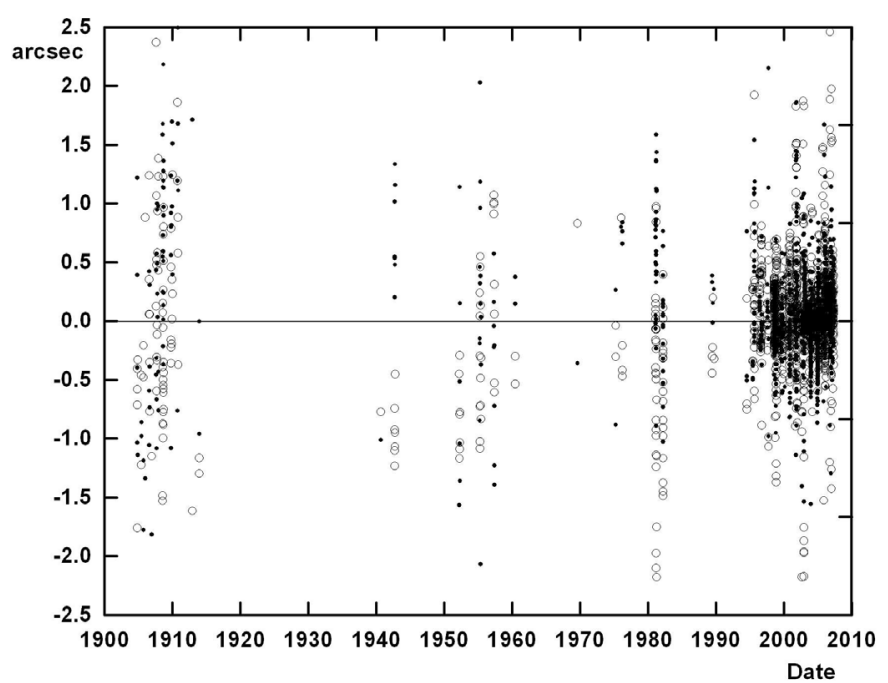

Fig. 1. Plots of residuals $\mathrm{O}-\mathrm{C}$ in right ascension (points) and declination (circles) for the whole set of used observations.

the equations of motion was carried out up to the final date of the ephemeris, October 22, 2027.

We used a least-squares procedure to fit the orbits to observations by adjusting the epoch state vector of the integrated orbit. Conditional equations were solved and corrections to the initial conditions were obtained; this process was then repeated. Improvement of the initial conditions was stopped when the corrections were 50 times less than their errors defined by a least-squares fit. After the process was completed, the last version of Chebyshev polynomials for the rectangular planetocentric coordinates of Phoebe was stored for subsequent ephemeris calculation.

At the preliminary least-squares fit of the orbit to observations, root mean squares (rms) of residuals in astrometric positions of Phoebe $\sigma_{d}^{(n)}$ were found for all the observations of each observatory $n$. In subsequent fits, weights were assigned to each observation made at observatory $n$ according to the value of $\sigma_{d}^{(n)}$.

For the observations made prior to 1940 we had to apply an additional correction of -0.75 arcsec to all right ascensions, as explained in Jacobson (2000). Although it is not strictly correct, we also applied the additional corrections of -0.75 arcsec to all right ascensions and declinations for the observations made prior to 1963 . Our tests confirmed that these corrections improve the fit, and force the mean value of residuals to be close to zero.

A summary of residual statistics for different observatories is given in Table 1. The mean values $\overline{r_{\alpha}}, \overline{r_{\delta}}$, and unweighted root mean squares $\sigma_{\alpha}, \sigma_{\delta}$ of residuals in right ascension and declination, are calculated for the observations made at each observatory. Note that all residuals in right ascension were multiplied by $\cos \delta$.

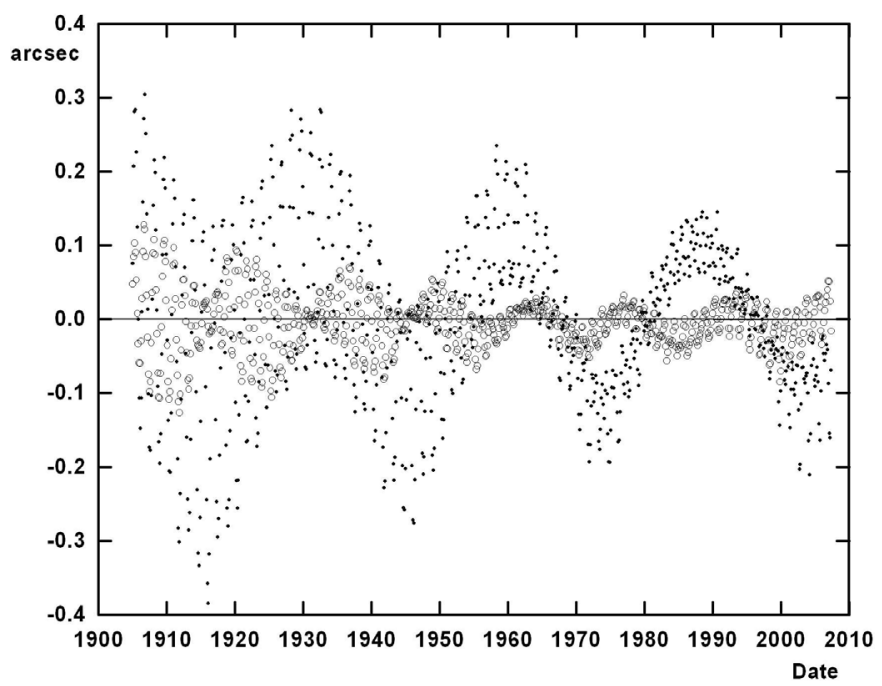

Fig. 2. Plots of JPL ephemeris deviations from our in right ascension (points) and declination (circles) on the period of observations of Phoebe.

Total residual statistics for all 1606 observations are given in Table 2. Figure 1 shows the $\mathrm{O}-\mathrm{C}$ residuals in right ascension and declination for all observations used.

To check our ephemeris, a comparison was made with the JPL ephemeris. The JPL ephemeris was generated online using the Horizons system (Giorgini et al. 1996).

For Phoebe, the ephemeris was generated in the time interval from 1905 to 2007, with a step of 50 days. The discrepancies between the JPL ephemeris and ours are shown in Fig. 2. These discrepancies are less than the observational errors seen in the majority of the observations, and may be accounted for by the different observation sets used at JPL and in our work. Note that in the Fig. 2 one can see some definitive periods in the differences.

We compared the two independent models of satellite motion as functions of the initial conditions of integration. For this we used the Horizons system to generate right ascension and declination values for Phoebe, in the interval 1905-2007 with a step of 50 days. Considering the JPL ephemeris as observations, we improved the initial conditions of integration using our calculating program. Residuals obtained in this way can be caused only by the differences in the two models of satellite motion, since there are no observational errors. In this case, residuals in right ascension and declination did not exceed $0.020^{\prime \prime}$. Rms residuals proved to be $0.008^{\prime \prime}$ in both right ascension and declination. These results show that the disagreement between the JPL and our models is significantly less than the errors in the available observations.

\section{Conclusions}

We have elaborated an ephemeris of Phoebe based on all groundbased observations available to date. In the theory of satellite motion, all necessary perturbations were taken into account, which provides precision of topocentric positions within $0.02^{\prime \prime}$. The real precision of the ephemeris is restricted by the accuracy of observations.

Comparison of the adopted model of satellite motion with that of JPL reveals that the precision of the adopted model is at least 20 times better than that of present-day observations. 
Presently, the dates for which an ephemeris of Phoebe may be calculated may be taken beginning from the first observation date in 1904 to 2027. Our estimate of real precision of the ephemeris in satellite topocentric position is not worse than $0.15^{\prime \prime}$.

The model of Phoebe's motion will be constantly updated as new observations become available. This will improve the ephemeris precision.

The ephemeris is available at: http: //www . imcce. fr/sat and http://www.sai.msu.ru/neb/nss/index.htm.

Acknowledgements. This work was supported by the Russian Foundation for Basic Research, project No. 06-02-16966-a.

\section{References}

Aoki, S., Soma, M., Kinoshita, H., \& Inoue, K. 1983, A\&A, 129, 263 Arlot, J.-E., Bec-Borsenberger, A., Fienga, A., \& Baron, N. 2003, A\&A, 411, 309

Belikov, M. V. 1993, Manuscripta geodaetica, 18, 182

Emelianov N. V. 1996, Ephemerides software of natural satellites, Annales de physique, PHESAT95 Workshop, Colloque C1, 21, C1-41

Emelyanov N. V. 2005, A\&A, 435, 1173

Emelyanov, N. V., Arlot, J.-E., Varfolomeev, M. I., et al. 2006, Cosm. Res., 44, 128

Feissel, M., \& Mignard, F. 1998, A\&A, 331, L33

Giorgini, J. D., Yeomans, D. K., Chamberlin, A. B., et al. 1996, BAAS, 28, 25.04 Jacobson, R. A. 2000, AJ, 120, 2679

Jacobson, R. A. 2004, AJ, 128, 492

Jacobson, R. A., Antreasian, P. G., Bordi, J. J., et al. 2006, AJ, 132, 2520

Shen, K. X., Harper, D., Qiao, R. C., et al. 2005, A\&A, 437, 1109 